\title{
Predictive Factors for Postoperative Renal Function After Off-Clamp, Non-Renorrhaphy Partial Nephrectomy
}

\section{Masaki Nakamura ( $\square$ masakin64@gmail.com )}

NTT Medical Center Tokyo

\section{Shuji Kameyama}

NTT Medical Center Tokyo

Taro Teshima

NTT Medical Center Tokyo

Yasushi Inoue

NTT Medical Center Tokyo

Tadashi Yoshimatsu

NTT Medical Center Tokyo

Yoshiki Ambe

NTT Medical Center Tokyo

Norihide Shirakawa

NTT Medical Center Tokyo

Hiroki Inatsu

NTT Medical Center Tokyo

Ryo Amakawa

NTT Medical Center Tokyo

Shunsuke Imai

NTT Medical Center Tokyo

Masashi Kusakabe

NTT Medical Center Tokyo

Teppei Morikawa

NTT Medical Center Tokyo

Yoshiyuki Shiga

NTT Medical Center Tokyo

\section{Research Article}

Keywords: Renal pedicle clamping, renorrhaphy, partial nephrectomy, estimated glomerular filtration rate, early recovery 
Posted Date: September 8th, 2021

DOl: https://doi.org/10.21203/rs.3.rs-871351/v1

License: (c) (i) This work is licensed under a Creative Commons Attribution 4.0 International License. Read Full License 


\section{Abstract}

Background: There is limited information on perioperative renal function during off-clamp, nonrenorrhaphy open partial nephrectomy.Therefore, this study aimed to clarify the chronological postoperative changes in renal function after off-clamp, non-renorrhaphy open partial nephrectomy.

Methods: Clinical records of 138 patients with renal tumors who underwent off-clamp, non-renorrhaphy open partial nephrectomy at our institution were reviewed. Off-clamp, non-renorrhaphy partial nephrectomy were performed using a soft coagulation system. Perioperativeestimated glomerular filtration rate (eGFR) preservation was calculated, and predictors were identified using multivariate regression analysis at 5 days, 1 month, and 3 months after surgery.

Results: The median operation time was 122 minutes, and the median volume of estimated blood loss was $155 \mathrm{~mL}$. The mean eGFR preservation at 5 days, 1 month, and 3 months after surgery was $95.3 \%$, $91.0 \%$, and $90.7 \%$, respectively. Multivariate regression analysis revealed that estimated blood loss was an independent predictor of perioperative eGFR preservation at 5 days and 1 month after surgery, while age was an independent predictor of perioperative eGFR preservation at 3 months after surgery.

Conclusion: Chronological changes in renal function after off-clamp, non-renorrhaphy open partial nephrectomy have been reported.Ourresults could bea reference in the era of robot-assisted partial nephrectomy.

\section{Introduction}

Partial nephrectomy has become the standard procedure for removing renal tumors. Robot-assisted partial nephrectomy (RAPN) has been established as a safe and minimally invasive procedure for tumors measuring $<40 \mathrm{~mm}[1-3]$. Renal pedicle clamping and renorrhaphy are included in laparoscopic partial nephrectomy in most institutes. However, these operative procedures cause postoperative complications, such as renal function impairment or pseudoaneurysm, at a certain rate [4].

Preservation of estimated glomerular filtration rate [eGFR] and no chronic kidney disease [CKD] upgrading are important outcomes after RAPN $[1,3,5,6]$. Although studies have reported eGFR preservation after on-clamp partial nephrectomy with renorrhaphy (open or laparoscopic), limited studies have reported that after off-clamp open partial nephrectomy without renorrhaphy. In particular, assessment of renal function impairment at very early time points ( $<3$ months after surgery) is lacking.

We retrospectively analyzed the perioperative data of 138 patients who underwent off-clamp open partial nephrectomy without renorrhaphy. The association between perioperative renal function preservation and tumor characteristics and patient characteristics was analyzed.

\section{Results}


Patient characteristics are described in Table 1. There were 92 (66.7\%) were male patients and $46(33.3 \%)$ female patients. The median age and tumor size were 63 years (range, 27-89 years) and $28 \mathrm{~mm}$ (range, 7-110 mm), respectively. Among the tumors, 41 (29.7\%) were > 50\% exophytic, 62 (44.9\%) were $<50 \%$ exophytic, and 35 (25.4\%) were entirely endophytic. The median nephrometry score was 7 (range, 4-10). 
Table 1

Patient and tumor characteristics

No. patients

138

$\operatorname{Sex}(\%)$

Male

$92(66.7)$

Female

46 (33.3)

Median age (range)

$63(27-89)$

Median BMI (range)

23.4

$(14.6-38.9)$

No. tumor laterality (\%)

Right

$69(50.0)$

Left

$69(50.0)$

Median tumor size, $\mathrm{mm}$ (range)

$28(7-110)$

Median RENAL nephrometry score (range)

$7(4-10)$

Exophytic/Endophytic properties

$>50 \%$ exophytic

$41(29.7)$

$<50 \%$ exophytic

$62(44.9)$

Entirely endophytic

$35(25.4)$

Location relative to the polar lines

Entirely above the upper or below the lower polar line

46 (33.3)

Lesion crosses polar line

64 (46.4)

$>50 \%$ of mass is across polar line or mass crosses the axial renal midline or mass is $\quad 28(20.3)$ entirely between the polar lines

Comorbidity, n (\%)

Hypertension

Diabetes mellitus

$22(15.9)$

Dyslipidemia

$17(12.3) 4$

BMI Body Mass Index. The RENAL nephrometry score is the sum of the sectional scores $(R, E, N$, and L). RCC Renal cell carcinoma,

The surgical results are described in Table 2. The median operation time was $122.5 \mathrm{~min}$. The median estimated blood loss was $155 \mathrm{~mL}$. There were no cases of conversion to nephrectomy or consequential 
renal hilum clamping. The mean eGFR preservation at 5 days, 1 month, and 3 months after surgery was $95.3 \%, 91.0 \%$, and $90.7 \%$, respectively. The relationship between age and perioperative eGFR decline was shown in Fig. 1A and 1B. Statistically significant reduction in eGFR was detected only in patients aged over 80 (Fig. 1A).

\section{Table 2}

Surgical results and complications

\begin{tabular}{|ll|}
\hline Median operative time, min (range) & $\mathbf{1 2 2 . 5}(\mathbf{5 6 - 1 9 9 )}$ \\
\hline Median volume of estimated blood loss, mL (range) & $155(5-1070)$ \\
\hline No. additional resections & 0 \\
\hline No. conversion to nephrectomy & 0 \\
\hline Intraoperative blood transfusion & 0 \\
\hline Postoperative blood transfusion & 0 \\
\hline Pathology (\%) & \\
\hline Clear cell RCC & $92(66.7)$ \\
\hline Papillary RCC & $13(9.4)$ \\
\hline Chromophobe RCC & $10(7.2)$ \\
\hline Angiomyolipoma & $17(12.3)$ \\
\hline Oncocytoma & $2(1.4)$ \\
\hline Others & $14(10.1)$ \\
\hline Mean eGFR preservation, \% (SD) & $95.3(14.9)$ \\
\hline Five days after surgery & $91.0(11.5)$ \\
\hline One month after surgery & $90.7(16.3$ \\
\hline Three months after surgery & \\
\hline eGFR estimated glomerular filtration ratio, RCC renal cell carcinoma & \\
\hline
\end{tabular}

Tumor size, R, N, RENAL score, and estimated blood loss were identified predictors of perioperative eGFR preservation at 5 days after surgery. Multivariate analysis revealed that estimated blood loss was an independent predictor of perioperative eGFR preservation (Table 3). 
Table 3

Predictors of perioperative eGFR preservation at 5 days after surgery. Univariate and multivariate analysis Univariate analysis Multivariate analysis

\begin{tabular}{|c|c|c|c|c|c|c|}
\hline & $\begin{array}{l}\text { Unstandardized } \\
\text { regression } \\
\text { coefficient B } \\
(95 \% \mathrm{Cl})\end{array}$ & $\begin{array}{l}\text { Standardized } \\
\text { regression } \\
\text { coefficient } \beta\end{array}$ & $\begin{array}{l}p- \\
\text { value }\end{array}$ & $\begin{array}{l}\text { Unstandardized } \\
\text { regression } \\
\text { coefficient B } \\
(95 \% \mathrm{Cl})\end{array}$ & $\begin{array}{l}\text { Standardized } \\
\text { regression } \\
\text { coefficient } \beta\end{array}$ & $\begin{array}{l}p- \\
\text { value }\end{array}$ \\
\hline $\mathrm{Age}^{\dagger}$ & $\begin{array}{l}-0.128(-0.332 \\
0.077)\end{array}$ & -0.105 & 0.219 & $\begin{array}{l}-0.174(-0.364 \\
0.017)\end{array}$ & -0.143 & 0.074 \\
\hline BMI & $\begin{array}{l}-0.470(-1.054 \\
0.114)\end{array}$ & -0.136 & 0.114 & & & \\
\hline $\begin{array}{l}\text { Tumour } \\
\text { size }^{\dagger}\end{array}$ & $\begin{array}{l}-0.218(-0.356 \\
-0.081)\end{array}$ & -0.261 & $0.02^{*}$ & $\begin{array}{l}-0.101(-0.247 \\
0.045)\end{array}$ & -0.121 & 0.172 \\
\hline $\mathrm{R}$ & $\begin{array}{l}-6.417(-11.209 \\
-1.625)\end{array}$ & -0.221 & $0.009^{*}$ & & & \\
\hline$E$ & $\begin{array}{l}-0.926(-2.487 \\
4.339)\end{array}$ & -0.046 & 0.592 & & & \\
\hline $\mathrm{N}^{+}$ & $\begin{array}{l}-3.752(-6.615 \\
-0.89)\end{array}$ & -0.217 & 0.011 & & & \\
\hline L & $\begin{array}{l}-2.435(-5.923 \\
1.054)\end{array}$ & -0.118 & 0.17 & & & \\
\hline $\begin{array}{l}\text { RENAL } \\
\text { score }^{\dagger}\end{array}$ & $\begin{array}{l}-1.819(-3.276 \\
-0.362)\end{array}$ & -0.207 & $0.015^{\star}$ & $\begin{array}{l}-1.112(-2.559 \\
0.335)\end{array}$ & -0.127 & 0.131 \\
\hline $\begin{array}{l}\text { Operative } \\
\text { time }\end{array}$ & $\begin{array}{l}-0.055(-0.132 \\
0.023)\end{array}$ & -0.118 & 0.168 & & & \\
\hline $\begin{array}{l}\text { eBlood } \\
\text { loss }^{\dagger}\end{array}$ & $\begin{array}{l}-0.024(-0.033 \\
-0.014)\end{array}$ & -0.377 & $\begin{array}{l}< \\
0.001^{*}\end{array}$ & $\begin{array}{l}-0.019(-0.03 \\
-0.008)\end{array}$ & -0.3536 & $0.001^{*}$ \\
\hline HTN & $\begin{array}{l}-3.198(-8.356, \\
1.96)\end{array}$ & -0.105 & 0.222 & & & \\
\hline DM & $\begin{array}{l}-4.427(-11.301 \\
2.446)\end{array}$ & -0.109 & 0.205 & & & \\
\hline $\mathrm{DL}$ & $\begin{array}{l}-1.052(-8.751 \\
6.647)\end{array}$ & -0.023 & 0.787 & & & \\
\hline
\end{tabular}

eGFR estimated glomerular filtration rate, BMI body mass index. eBlood loss estimated blood loss, $\mathrm{R}$ Radius, E exophytic and endophytic properties, $\mathrm{N}$ Nearness of the tumor to the collecting system or sinus, $\mathrm{L}$ location relative to the upper and lower polar lines, (R,E,N, and $\mathrm{L}$ were scored according to RENAL nephrometry scoring system), HTN hypertension, DM diabetes mellitus, DL dyslypidemia, Cl confidential interval, ${ }^{\dagger}$ These factors were put in multivariate regression analysis, ${ }^{*} p$-value $<0.05$ was considered statistically significant. 
Tumor size, R, RENAL score, and estimated blood loss were identified as predictors of perioperative eGFR preservation at 1 month after surgery. Multivariate analysis revealed that age, tumor size, and estimated blood loss were independent predictors of the eGFR preservation (Table 4). 
Table 4

Predictors of perioperative eGFR preservation at 1 month after surgery. Results of univariate and multivariate analysis.

\begin{tabular}{|c|c|c|c|c|c|c|}
\hline & \multicolumn{3}{|c|}{ Univariate analysis } & \multicolumn{3}{|c|}{ Multivariate analysis } \\
\hline & $\begin{array}{l}\text { Unstandardized } \\
\text { regression } \\
\text { coefficient B } \\
(95 \% \mathrm{Cl})\end{array}$ & $\begin{array}{l}\text { Standardized } \\
\text { regression } \\
\text { coefficient } \beta\end{array}$ & $\begin{array}{l}p- \\
\text { value }\end{array}$ & $\begin{array}{l}\text { Unstandardized } \\
\text { regression } \\
\text { coefficient B } \\
(95 \% \mathrm{Cl})\end{array}$ & $\begin{array}{l}\text { Standardized } \\
\text { regression } \\
\text { coefficient } \beta\end{array}$ & $\begin{array}{l}p- \\
\text { value }\end{array}$ \\
\hline $\mathrm{Age}^{\dagger}$ & $\begin{array}{l}-0.118(-0.275 \\
0.039)\end{array}$ & -0.127 & 0.140 & $\begin{array}{l}-0.158(-0.307 \\
-0.008)\end{array}$ & -0.17 & $0.039^{*}$ \\
\hline Sex & $\begin{array}{l}1.08(-3.08 \\
5.239)\end{array}$ & 0.044 & 0.608 & & & \\
\hline BMI & $\begin{array}{l}-0.167(-0.618 \\
0.285)\end{array}$ & -0.063 & 0.467 & & & \\
\hline $\begin{array}{l}\text { Tumour } \\
\text { size }^{\dagger}\end{array}$ & $\begin{array}{l}-0.182(-0.287 \\
-0.077)\end{array}$ & -0.283 & $0.001^{*}$ & $\begin{array}{l}-0.121(-0.235 \\
-0.006)\end{array}$ & -0.187 & $0.039^{\star}$ \\
\hline $\mathrm{R}$ & $\begin{array}{l}-5.612(-9.276 \\
-1.948)\end{array}$ & -0.252 & 0.003 & & & \\
\hline$E^{\dagger}$ & $\begin{array}{l}0.441(-2.204 \\
3.085)\end{array}$ & 0.028 & 0.742 & & & \\
\hline $\mathrm{N}$ & $\begin{array}{l}-2.187(-4.425 \\
0.052)\end{array}$ & -0.164 & 0.055 & & & \\
\hline L & $\begin{array}{l}-1.597(-4.302, \\
1.107)\end{array}$ & -0.1 & 0.245 & & & \\
\hline $\begin{array}{l}\text { RENAL } \\
\text { score }\end{array}$ & $\begin{array}{l}-1.277(-2.404 \\
-0.15)\end{array}$ & -0.189 & $0.027^{*}$ & $\begin{array}{l}-0.769(-1.902 \\
0.364)\end{array}$ & -0.114 & 0.182 \\
\hline $\begin{array}{l}\text { Operative } \\
\text { time }\end{array}$ & $\begin{array}{l}-0.045(-0.105 \\
0.015)\end{array}$ & -0.126 & 0.144 & & & \\
\hline $\begin{array}{l}\text { eBlood } \\
\text { loss }^{\dagger}\end{array}$ & $\begin{array}{l}-0.015(-0.023 \\
-0.007)\end{array}$ & -0.306 & 0.000 & $\begin{array}{l}-0.01(-0.018 \\
-0.002)\end{array}$ & -0.209 & $0.018^{\star}$ \\
\hline HTN & $\begin{array}{l}-2.576(-6.567 \\
1.415)\end{array}$ & -0.109 & 0.204 & & & \\
\hline DM & $\begin{array}{l}-1.976(-7.393 \\
3.442)\end{array}$ & -0.062 & 0.472 & & & \\
\hline $\mathrm{DL}$ & $\begin{array}{l}-2.421(-8.496 \\
3.653)\end{array}$ & -0.068 & 0.432 & & & \\
\hline
\end{tabular}




\section{Univariate analysis}

Multivariate analysis

eGFR estimated glomerular filtration rate, BMI body mass index. eBlood loss estimated blood loss, $\mathrm{R}$ Radius, E exophytic and endophytic properties, $\mathrm{N}$ Nearness of the tumor to the collecting system or sinus, $\mathrm{L}$ location relative to the upper and lower polar lines, (R,E,N, and $\mathrm{L}$ were scored according to RENAL nephrometry scoring system), HTN hypertension, DM diabetes mellitus, DL dyslypidemia, Cl confidential interval, ${ }^{\dagger}$ These factors were put in multivariate regression analysis, $\mathrm{Cl}$ confidence interval, * $p$-value $<0.05$ was considered statistically significant.

Age, tumor size, $\mathrm{R}, \mathrm{N}$, and estimated blood loss were identified as predictors of perioperative eGFR preservation at 3 months after surgery. Multivariate analysis revealed that age was an independent predictor of perioperative eGFR preservation (Table 5). 
Table 5

Predictors of perioperative eGFR preservation at 3 months after surgery. Results of univariate and multivariate analysis.

\begin{tabular}{|c|c|c|c|c|c|c|}
\hline & \multicolumn{3}{|c|}{ Univariate analysis } & \multicolumn{3}{|c|}{ Multivariate analysis } \\
\hline & $\begin{array}{l}\text { Unstandardized } \\
\text { regression } \\
\text { coefficient B } \\
(95 \% \mathrm{Cl})\end{array}$ & $\begin{array}{l}\text { Standardized } \\
\text { regression } \\
\text { coefficient } \beta\end{array}$ & $\begin{array}{l}p- \\
\text { value }\end{array}$ & $\begin{array}{l}\text { Unstandardized } \\
\text { regression } \\
\text { coefficient B } \\
(95 \% \mathrm{Cl})\end{array}$ & $\begin{array}{l}\text { Standardized } \\
\text { regression } \\
\text { coefficient } \beta\end{array}$ & $\begin{array}{l}p- \\
\text { value }\end{array}$ \\
\hline $\mathrm{Age}^{\dagger}$ & $\begin{array}{l}-0.228(-0.449 \\
0.008)\end{array}$ & -0.173 & $0.043^{*}$ & $\begin{array}{l}-0.277(-0.494 \\
-0.059)\end{array}$ & -0.209 & $0.013^{*}$ \\
\hline Sex & $\begin{array}{l}-1.981(-7.853 \\
3.89)\end{array}$ & -0.057 & 0.506 & & & \\
\hline BMI & $\begin{array}{l}-0.073(-0.727 \\
0.580)\end{array}$ & -0.019 & 0.824 & & & \\
\hline $\begin{array}{l}\text { Tumour } \\
\text { size }^{\dagger}\end{array}$ & $\begin{array}{l}-0.182(-0.334 \\
-0.03)\end{array}$ & -0.199 & $0.019 *$ & $\begin{array}{l}-0.277(-0.494 \\
0.039)\end{array}$ & -0.139 & 0.133 \\
\hline $\mathrm{R}$ & $\begin{array}{l}-6.019(-11.274, \\
-0.764)\end{array}$ & -0.191 & $0.025^{\star}$ & & & \\
\hline E & $\begin{array}{l}0.706(-3.014 \\
4.426)\end{array}$ & 0.032 & 0.708 & & & \\
\hline $\mathrm{N}$ & $\begin{array}{l}-3.058(-6.21 \\
0.094)\end{array}$ & -0.162 & 0.057 & & & \\
\hline L & $\begin{array}{l}-1.474(-5.292 \\
2.344)\end{array}$ & -0.065 & 0.446 & & & \\
\hline $\begin{array}{l}\text { RENAL } \\
\text { score }^{+}\end{array}$ & $\begin{array}{l}-1.472(-3.075 \\
-0.13)\end{array}$ & -0.154 & 0.071 & $\begin{array}{l}-1.113(-2.764 \\
0.537)\end{array}$ & -0.116 & 0.184 \\
\hline $\begin{array}{l}\text { Operative } \\
\text { time }\end{array}$ & $\begin{array}{l}0.01(-0.075 \\
0.095)\end{array}$ & 0.02 & 0.817 & & & \\
\hline $\begin{array}{l}\text { eBlood } \\
\text { loss }^{\dagger}\end{array}$ & $\begin{array}{l}-0.014(-0.025 \\
-0.003)\end{array}$ & -0.206 & $0.015^{\star}$ & $\begin{array}{l}-0.009 \\
0.003)\end{array}$ & -0.128 & 0.156 \\
\hline HTN & $\begin{array}{l}-3.94(-9.549 \\
1.67)\end{array}$ & -0.118 & 0.167 & & & \\
\hline DM & $\begin{array}{l}-4.882(-12.368 \\
2.604)\end{array}$ & -0.11 & 0.199 & & & \\
\hline DL & $\begin{array}{l}-1.584(-9.969 \\
6.8)\end{array}$ & -0.032 & 0.709 & & & \\
\hline
\end{tabular}


eGFR estimated glomerular filtration rate, BMI body mass index. eBlood loss estimated blood loss, $\mathrm{R}$ Radius, E exophytic and endophytic properties, N Nearness of the tumor to the collecting system or sinus, $L$ location relative to the upper and lower polar lines, $(R, E, N$, and $L$ were scored according to RENAL nephrometry scoring system), HTN hypertension, DM diabetes mellitus, DL dyslypidemia, $\mathrm{Cl}$ confidential interval, ${ }^{\dagger}$ These factors were put in multivariate regression analysis, ${ }^{*} p$-value $<0.05$ was considered statistically significant.

\section{Discussion}

We have reported on perioperative renal function preservation during off-clamp, non-renorrhaphy open partial nephrectomy within 3 months after surgery.

The advantage of the off-clamp technique in perioperative renal function preservation is controversial. Wen et al. have reported less decrease in renal function in off-clamp surgery compared to that in onclamp surgery (weighted mean difference: $4.81 \mathrm{~mL} / \mathrm{min} / 1.73 \mathrm{~m}^{2} ; 95 \%$ confidence interval: 3.53-6.08; $\mathrm{p}$ 0.00001 ) [9]. Meanwhile, several studies have failed to show the advantages of off-clamp surgery in eGFR preservation over clamping surgery in pneumoperitoneum settings [10-13].

AS for the advantage of renorrhaphy, the non-renorrhaphy technique failed to benefit the preservation of perioperative renal function for $\geq \mathrm{T} 1 \mathrm{~b}$ renal tumors in open partial nephrectomy compared with the cold ischemia technique [14]. In this study, the renal function was analyzed at 4 and 6 months after surgery. However, studies comparing single-layered and double-layered renorrhaphy have shown the benefits of single-layered renorrhaphy in eGFR preservation $[4,15,16]$. In these analyses, eGFR was assessed between the date of discharge and 1 month postoperatively. Considering these findings, it is possible that omission of renorrhaphy preserves renal function during the early postoperative period.

The RENAL score correlates with perioperative reduction in renal function during on-clamp partial nephrectomy [17]. In this study, split renal function was measured using diethylene triamine penta-acetic acid scintigraphy, which showed a significant decrease in ipsilateral renal function 6 months after surgery, with no significant change thereafter [17].

There are limited data on perioperative renal function during combined off-clamp and non-renorrhaphy partial nephrectomy. We have recently reported the surgical results of off-clamp, non-renorrhaphy open partial nephrectomy for $\geq T 1 \mathrm{~b}$ renal tumors [8]. The perioperative eGFR preservation at 1 month and 3 months after surgery was $88.9 \%$ and $87.3 \%$, respectively [8]. In laparoscopic or robotic surgeries, perioperative eGFR preservation was $96.9-100 \%$ for highly selected patients $[18,19]$. In our study, the eGFR preservation at 5 days, 1 month, and 3 months after surgery was $95.3 \%, 91.0 \%$, and $90.7 \%$, respectively. We believe our report will add some knowledge on the chronological recovery of renal function after off-clamp, non-renorrhaphy partial nephrectomy. 
Estimated blood loss was an early predictor of perioperative eGFR preservation, but was replaced by age at 3 months after surgery. Within 1 month after surgery, we assumed that damage of renal parenchyma by soft coagulation directly contributed to the decrease in the eGFR. Impairment of the potential for renal function recovery by age may affect eGFR preservation $>3$ months after surgery [20].

This study has some limitations. First, the study was a retrospective study. Second, the postoperative eGFR was analyzed only within 3 months after surgery.

In conclusion, we have analyzed perioperative changes in renal function after off-clamp, non-renorrhaphy open partial nephrectomy until 3 months after surgery. The perioperative eGFR preservation rates of $95.3 \%, 91.0 \%$, and $90.7 \%$ at 5 days, 1 month, and 3 months after surgery, respectively, reported in our study may be used as reference in the era of RAPN.

\section{Methods}

\section{Patients}

Among 220 patients with renal tumors who underwent off-clamp, non-renorrhaphy open partial nephrectomy at our institute between 2013 and 2020, those with postoperative complications of urinoma or incomplete data were excluded. Therefore, clinical records of 138 patients were retrospectively analyzed.

\section{Surgical Techniques}

Complexity of renal tumors were analyzed according to R-E-N-A-L nephrometry scoring system [7]. Briefly, (R)adius (tumor size as maximal diameter), (E)xophytic and endophytic properties, (N)earness of the tumor to the collecting system or sinus, and (L)ocation relative to the upper and lower polar lines were scored on a 1, 2 or 3-point scale. RENAL score represents sum of R, E, N, and L scores. All patients underwent open partial nephrectomy retroperitoneally. Renal pedicle was not secured, and renal pedicle clamping and cortical renorrhaphy were omitted. We used monopolar SOFT COAG (VIO300D, ERBE, Germany) with a normal saline drip for hemostasis. Surgical techniques have been described previously [8]. In partial nephrectomy, the renal parenchyma was repeatedly separated bluntly using a spatulashaped tip of a monopolar device, followed by soft coagulation of the separated renal parenchyma and bleeding blood vessels. To minimize blood loss, tumor resection was advanced by millimeter unit. Resection beds were sutured with 4-0 VICRYL ${ }^{\circledR}$ when the collecting system was opened. Urine leakage was ruled out by intravenous injection of indigo carmine solution. TachoSil $\circledast$ was placed on the resection surface to ensure hemostasis.

\section{Assessment of renal function and perioperative reduction in renal function}


The eGFR was calculated using the Eq. $186 \times(\text { Creatinine/88.4 })^{-1.154} \times(\text { Age })^{-0.203} \times(0.742$ if female $)$. Perioperative eGFR preservation at 5 days, 1 month, and 3 months after surgery was calculated as postoperative eGFR/preoperative eGFR $\times 100(\%)$.

\section{Statistical analysis}

The Student's t-test was used to analyze continuous variables. Univariate and multivariate regression analyses were performed to identify predictors of postoperative eGFR preservation. Statistical significance was set at $p<0.05$. All statistical analyses were performed using the SPSS version 24 .

\section{Declarations}

\section{Author contributions}

Data collection: M.N., Y.A.; Writing - original draft preparation: M.N.; Writing - review and editing: M.N., S.K., T.T., Y.I., T.Y., Y.A., N.S., H.I., R.A., S.I., M.K., T.M., Y.S.; Supervision: S.K., Y.S.

\section{Statement of Ethics}

This study was approved by the Ethics Committee of NTT Medical Center, Tokyo (ID: 20-198), and was conducted in accordance with the Helsinki Declaration.

Informed consent was obtained in the form of opt-out on the web-site (https://www.nmct.ntteast.co.jp/aboutus/ethic/research/). Those who rejected were excluded.

\section{Conflict ofInterest Statement}

The authors declare no competing interests.

\section{Funding Sources}

This research received no specific grantfrom any funding agency in the public, commercial, or not-for-profit sectors.

\section{Data Availability Statement}

All data generated or analysed during this study are included in this article. Further enquiries can be directed to the corresponding author.

\section{References}

1. Sri D, Thakkar R, Patel HR,et al(2020) Robotic-assisted partial nephrectomy (RAPN) and standardization of outcome reporting: a prospective, observational study on reaching the "Trifecta 
and Pentafecta". J Robot Surg doi: 10.1007/s11701-020-01141-z

2. Grivas N, Kalampokis N, Larcher A,et al (2019) Robot-assisted versus open partial nephrectomy: comparison of outcomes. A systematic review. Minerva UrolNefrol71:113-120. doi: 10.23736/S0393-2249.19.03391-5

3. Furukawa J,Kanayama H, Azuma H, et al (2020) 'Trifecta' outcomes of robot-assisted partial nephrectomy: a large Japanese multicentre study. Int J Clin Oncol25:347-353. doi: 10.1007/s10147019-01565-0

4. Bertolo R, Campi R, Mir MC,et al (2019) Systematic review and pooled analysis of the impact of renorrhaphy techniques on renal functional outcome after partial nephrectomy. Eur Urol Oncol2:572575 doi: 10.1016/j.euo.2018.11.008

5. Bravi CA, Larcher A, Capitanio U,et al(2021) Perioperative outcomes of open, laparoscopic, and robotic partial nephrectomy: a prospective multicenter observational study (the Record 2 project). Eur Urol Focus 7:390-396. doi: 10.1016/j.euf.2019.10.013

6. Minervini A, Campi R, Lane BR,et al (2020) Impact of resection technique on perioperative outcomes and surgical margins after partial nephrectomy for localized renal masses: a prospective multicenter study. J Urol203:496-504. doi: 10.1097/JU.0000000000000591

7. A. Kutikov and R. G. Uzzo. (2009) The R.E.N.A.L. nephrometry score: a comprehensive standardized system for quantitating renal tumor size, location and depth. J Urol, vol. 182, no. 3, pp. 844-53, doi: 10.1016/j.juro.2009.05.035.

8. Nakamura M,Ambe Y, Teshima T,et al.(2021) Assessment of surgical outcomes of off-clamp open partial nephrectomy without renorrhaphy for $\geq T 1 b$ renal tumours. Int J Clin Oncol. doi:

10.1007/s10147-021-01966-0

9. Deng W, Liu X, Hu J, et al (2018) Off-clamp partial nephrectomy has a positive impact on short- and long-term renal function: a systematic review and meta-analysis. BMC Nephrol19:188. doi:

10.1186/s12882-018-0993-3

10. Antonelli A, Veccia A, Francavilla S,et al (2019) On-clamp versus off-clamp robotic partial nephrectomy: a systematic review and meta-analysis. Urologia86:52-62. doi:

$10.1177 / 0391560319847847$

11. Anderson BG,Potretzke AM, Du K,et al (2019) Comparing off-clamp and on-clamp robot-assisted partial nephrectomy: a prospective randomized trial. Urology126:102-109. doi:

10.1016/j.urology.2018.11.053

12. Kreshover JE, Kavoussi LR, Richstone L (2013) Hilar clamping versus off-clamp laparoscopic partial nephrectomy for T1b tumors. CurrOpinUrol23:399-402 doi: 10.1097/MOU.0b013e3283632115

13. Anderson BG, Potretzke AM, Du K, et al (2018) Off-clamp robot-assisted partial nephrectomy does not benefit short-term renal function: a matched cohort analysis. J Robot Surg12:401-407. doi: $10.1007 /$ s11701-017-0745-6

14. Takagi T, Kondo T, Omae K, et al (2015) Assessment of surgical outcomes of the non-renorrhaphy technique in open partial nephrectomy for $\geq T 1 b$ renal tumors. Urology86:529-533. doi: 
10.1016/j.urology.2015.05.018

15. Bahler CD, Cary KC, Garg S,et al (2015) Differentiating reconstructive techniques in partial nephrectomy: a propensity score analysis. Can J Urol22:7788-7796.

16. Bahler CD,Dube HT, Flynn KJ,et al (2015) Feasibility of omitting cortical renorrhaphy during robotassisted partial nephrectomy: a matched analysis. J Endourol29:548-555. doi:

$10.1089 /$ end.2014.0763

17. Kwon T, Jeong IG, Ryu J, et al (2015) Renal function is associated with nephrometry score after partial nephrectomy: a study using diethylene triamine penta-acetic acid (DTPA) renal scanning. Ann Surg Oncol22 Suppl 3:S1594-S1600. doi: 10.1245/s10434-015-4500-9

18. Tohi Y, Makita N, Suzuki I,et al (2018) “Off-clamp, non-renorrhaphy robot-assisted partial nephrectomy: an initial experience in a single institution. Hinyokika Kiyo64:323-327. doi: 10.14989/ActaUrolJap_64_8_323

19. Simone G, Papalia R, Guaglianone S, et al (2012) 'Zero ischaemia', sutureless laparoscopic partial nephrectomy for renal tumours with a low nephrometry score. BJU Int110:124-130. doi: 10.1111/j.1464-410X.2011.10782.x

20. Denic A, Glassock RJ, Rule AD (2016) Structural and functional changes with the aging kidney. Adv Chronic Kidney Dis23:19-28. doi: 10.1053/j.ackd.2015.08.004

\section{Figures}

A

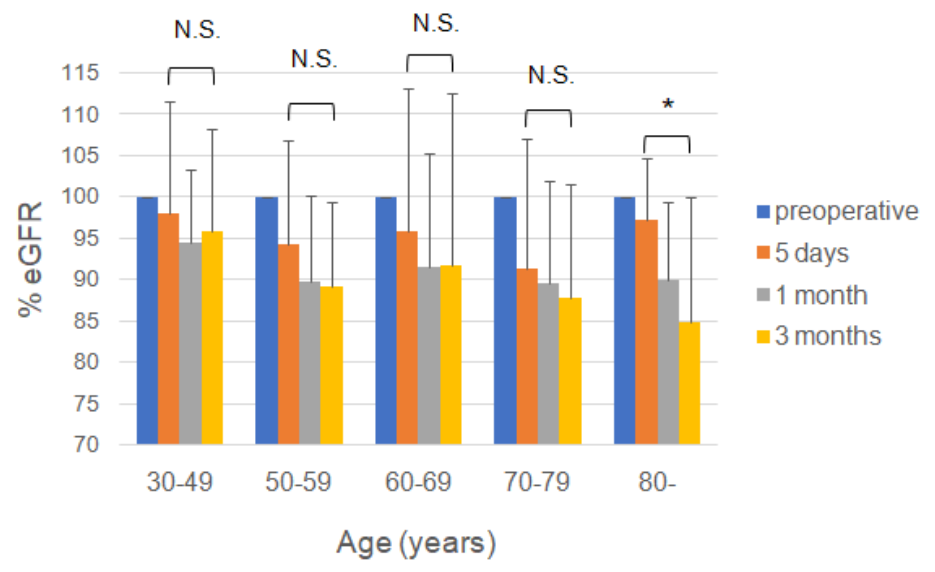

B

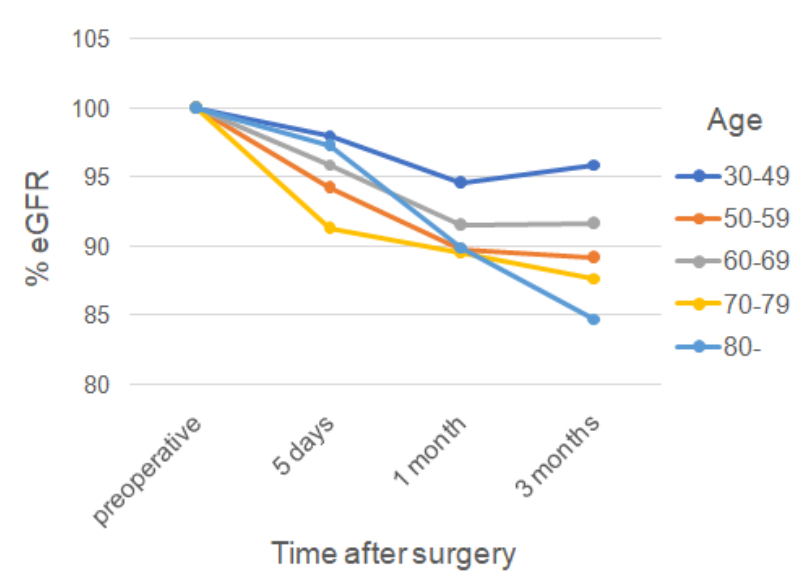

\section{Figure 1}

A) Relationship between age and eGFR change was shown. N.S. not significant. ${ }^{*} p<0.05$. B) Chronological change of eGFR (mean) by age are plotted. 